\title{
Evaluation of Lipid Peroxidation and Antioxidants' Status in Metabolic Syndrome
}

\author{
Shrestha $\mathrm{S}^{1}$, Chandra $\mathrm{L}^{2}$, Aryal $\mathrm{M}^{3}$, Das $\mathrm{BKL}^{4}$, Pandey $\mathrm{S}^{5}$, Baral $\mathrm{N}^{6}$
}

1,4-6 Department of Biochemistry,

B.P. Koirala Institute of Health Sciences, Dharan, Nepal

${ }^{2}$ Department of Biochemistry,

Maulana Azad Medical College, New Delhi, India

${ }^{3}$ Department of Biochemistry,

Kathmandu University School of Medical Sciences,

Dhulikhel, Nepal

\author{
ABSTRACT \\ Background
}

Metabolic syndrome is a constellation of physical conditions and metabolic abnormalities, commonly occurring together, that increases an individual's risk for development of type 2 diabetes mellitus and cardiovascular diseases. Oxidative stress is associated with diabetes, hypertension and other cardiovascular diseases while the role of oxidative stress in pathogenesis of MS is not clearly defined.

\section{Objectives}

The study aims to find out the prevalence of metabolic syndrome in faculty and staff members at BP Koirala Institute of Health Sciences, Dharan, Nepal and to evaluate oxidative stress levels in subjects with metabolic syndrome.

\section{Methods}

118 healthy participants working at B. P. Koirala Institute of Health Sciences, Dharan, Nepal were selected at random for this cross-sectional study and blood samples were collected for the estimation of the following biochemical analytes; fasting glucose; triglycerides; total cholesterol; high density lipoprotein cholesterol; Albumin; uric acid; Bilirubin; Malondialdehyde; Catalase; Glutathione peroxidase; Superoxide Dismutase; Glutathione; vitamin C; and lastly vitamin E.

Dr Lal Chandra

Department of Biochemistry,

Maulana Azad Medical College, New Delhi, India

Email. Ichandra70@yahoo.com

Kathmandu Univ Med J 2010;9(32):382-6

Results

In this cross-sectional study, $39 \%$ subjects were diagnosed with metabolic syndrome , particularly in sedentary subjects. There was no difference in oxidative stress except significant rises in serum uric acid levels and catalase activity in subjects diagnosed with metabolic syndrome .

\section{Conclusion}

The prevalence of metabolic syndrome is higher without oxidative stress in this study, which suggests that oxidative stress does not contribute to the pathogenesis of MS in otherwise healthy subjects.

\section{Key Words}

antioxidants, lipid peroxidation, metabolic syndrome, oxidative stress 


\section{INTRODUCTION}

Metabolic syndrome (MS) is a constellation of physical conditionsandmetabolicabnormalitieswhichincreasean individual'sriskofdevelopingtype2diabetesmellitusand cardiovasculardiseases.UsingthecriteriaoftheNational Cholesterol Education Programme Adult Treatment Panel III (NCEP ATPIII), the prevalence of the MS in U.S.adults' $\geq 20$ years ofagewasrecentlyestimated to be 23.7\%. 'LittleisknownabouttheprevalenceoftheMSin south-east Asians particularly in Nepal. 2,3

Oxidative stress is an imbalance between tissue, free radicals, reactiveoxygenspecies(ROS), andantioxidants system. The imbalance which causes oxidative stress is caused bye highly reactive molecules with unpaired electrons, that bind with nearby molecules leading to oxidative damage. Antioxidants prevent the series of reactions that generate free radicals or neutralises them. ${ }^{4}$ Oxidativestressisassociated withobesity-related conditions such as diabetes, hypertension and other cardiovasculardiseases.Thesediseaseshavebeen proven to have a direct association with MS.

ThisstudyaimstorevealthestatusofMSinfacultyandstaff members of B. P. Koirala Institute of Health Sciences, to enable the prevention of the disease; to promote overallhealthbychangingpeople'slifestyles;andthrough pharmacologicalinterventionifandwhen required.The study also aims to investigate lipid peroxidation and variousnon-enzymaticandtheenzymaticantioxidants status in MS cases.

\section{METHODS}

With the approval of the B.P. Koirala Institute of Health SciencesResearchCommittee, 118 healthyparticipants (98 male and 20 female) were randomly selected. The participants were not receiving antioxidant vitamin supplementation.Anthropometricmeasurements,blood pressure, ethnicity and personal habits were recorded in a pre-designed pro forma from each respondent. Bloodsamplesweretakeninthemorningafter 12-hour overnightfasting.Serum, plasmaandErythrocytelysate were preparedand storedat $-20^{\circ} \mathrm{Cuntiluse.Plasmared}$ cellswere washed with normal saline threetimes, then redbloodcellswerelysed withfourtimesitsvolumewith ice-chilled,distilledwater.Asaresult,Erythrocytelysate formed, and wasseparatedbycentrifugingat 10,000 rpm for 15 minutes at $4^{\circ} \mathrm{C}$.

\section{Definition of metabolic syndrome}

Metabolic syndrome was diagnosed according to the NCEP ATP III criteria. ${ }^{1}$ According to this criteria, the diagnosisofmetabolicsyndromewasestablishedwhen threeormoreofthefollowing riskfactorswere present; waistcircumference $>102 \mathrm{cminmenand}>88 \mathrm{cminwomen}$; fastingglucose $\geq 110 \mathrm{mg} /$ dlandtriglycerides $\geq 150 \mathrm{mg} / \mathrm{dl}$ in eithersex: HDL cholesterol $<50 \mathrm{mg} / \mathrm{dl}$ in women and $<40 \mathrm{mg} /$ dlinmen:bloodpressure-systolicbloodpressure (SBP) $\geq 130 \mathrm{~mm} \mathrm{Hg}$, diastolic blood pressure (DBP) $\geq 85 \mathrm{~mm} \mathrm{Hg}$ or use of antihypertensive medications.

\section{Biochemical parameters}

The biochemical parameters such as fasting glucose, totalcholesterol,triglycerides, highdensitylipoprotein (HDL-C), low density lipoprotein (LDL-C), uric acid, Albumin and Bilirubin were analysed from the serum on Vitalab Selectra-MERCK Clinical Chemistry Analyzer. Plasma concentration of lipid peroxidation productMalondialdehyde(MDA)wasestimated bythe measurementofthiobarbituricacidreactivesubstanceby the method of Yagi et al. ${ }^{5}$ Erythrocyte Catalase (CAT) wasassayedcolorimetricallyasmicromolesofhydrogen peroxideconsumedperminutepermilligramhaemoglobin as described by Sinha et al. ${ }^{6}$ Erythrocyte Superoxide Dismutase (SOD) was assayed in erythrocyte lysate by themethod ofKakkaretal.Thiswas based on inhibition oftheformationofNicotinamideAdenineDinucleotide, Phenazine Methosulfate and amino blue Tetrazolium Formazan.Asingleunitofenzymewasexpressedas $50 \%$ inhibition of NBT (Nitroblue Tetrazolium) reduction/ $\mathrm{min} / \mathrm{gm} \mathrm{Hb}^{7}$.ErythrocyteGlutathionePeroxidase (GPX) activitywasassayedinerythrocytelysatebythemethod described by Rotrucketal. ${ }^{8}$ asmicrogram ofGluthatione (GSH) consumed per minute per gram haemoglobin. ${ }^{8}$ Glutathione (GSH) in whole blood was determined by the method of Beutler et al. ${ }^{9}$ Plasma vitamin C was estimatedbymethoddependingonthereductionofferric ion ( $1 \%$ Ferric chloride) to ferrous ion by ascorbic acid present in protein free filtrate. It was reacted with $0.5 \%$ $\alpha, \alpha^{\prime}$-Dipyridyl to form a red-orange $\alpha, \alpha^{\prime}$-Dipyridyl complexmeasuredspectrophotometricallyat $520 \mathrm{~nm} .{ }^{10}$ PlasmavitaminEwasestimatedbythemethodinwhich $\alpha$-Tocopherolextractedinpetroleumetherisoxidizedto tocopherylquininebyferricchlorideandresultantferrous ion iscomplexed withethanolic $\alpha, \alpha^{\prime}$-Dipyridyl (0.2\%in ethanol)toproducearedcolouredcompound whichwas measuredspectrophotometricallyat520nmandexpressed as mg/dl. ${ }^{11}$ Haemoglobin $(\mathrm{Hb})$ in both wholebloodand erythrocytelysatewasmeasuredasdescribedbyDrabkin and Austin. ${ }^{12}$

\section{Statistical analyses}

Datawas analysedusing SPSS-11.5statistical package. Studentst'testandchi-squaretesting was used.Values were expressed in mean SD, and differences were considered significant at $p<0.05$. 


\section{RESULTS}

In this cross-sectional study, the prevalence of MS was 39\%.The distributions of MSand NMS (Non-metabolic syndrome)withsex,ethnicity, lifestyle,exercise,smoking, alcoholintakeisshowninTable-1.Theassociation ofMS with lifestyle and exercise was statistically significant, whichindicated thathigherincidencesofMSwerefound in those with a sedentary lifestyle and non exercising subjects.

The comparison of anthropometric measurements, componentsofMSandotherbiochemicalparametersin NMS and MS are shown in Table-2. All the components of MS were highly significant $(p<0.001)$. In addition MS Body Mass Index (BMI) than NMS group which was statistically significant. Total cholesterol and LDL werehigherinMSgroupascompared toNMSgroup.But differenceinLDLwashighlysignificantbetweentheNMS and MS group $(p<0.001)$.

Thecomparisonofantioxidantsandlipidperoxidationin MSgroupandNMSgroupareshowninTable-3.Plasma MDA, Erythrocyte GPx and CAT activity were slightly increased while SOD was decreased in MS group as compared to NMS but was statistically not significant. Uricacid $(p=0.014)$ and Catalaseactivity $(p=0.018)$ were significantly higher in MS group as compared to NMS.

\section{DISCUSSION}

Inthepastfewyearstherehasbeengrowinginterestinthe phenomenonofriskfactorclustering thatincreasesthe "globalrisk"foratheroscleroticcardiacvasculardiseases. One pattern of thisclustering is amplified by Metabolic Syndrome, labeledassuchbecausecardiovasculardiseases risk factors that make up this pattern appear to be of metabolicorigin.Moreoverduringthepastfewyears, a largedeclineincardiovasculardiseasemortalityhasbeen experiencedinthewestandsubstantialincreaseshave beenexperiencedinthedevelopingcountries. ${ }^{16}$ These trends are expected to continue, with the developing countriesexperiencing doubleburden ofboth preand posttransitionaldisease.Atthesametimeprevalenceof MSalso substantially increasesand varies according to thepopulationconsideredranging from $8.8 \%$ to $14.3 \%$ in Europe and $22.6 \%$ to $23.7 \%$ in United States. ${ }^{1,16} \mathrm{In}$ present study the prevalence of metabolic syndrome (39\%) is higher than developed countries like Europe and United States, indicating that it is a syndrome not restricted to affluent countries only. ${ }^{1}$

The present investigations have established that the antioxidantissubnormalinsubjectexhibitingMS.Ofthe five criteria of MS defined in NCEP/ATP III four (notably hypertriglyceridemia,hypertension, hyperglycemiaand abdominalobesity)areindependentlycharacterized by elevated systemic oxidative stress. ${ }^{13}$

Malondialdehyde is produced by peroxidative decomposition of polyunsaturated lipid. It is used as markeroffreeradicaltissuedamageandoxidativestress. In the present study MDA level was not statistically significant.ButinsimilarstudybyRomeroFGetal,there was significantly increased MDA level in MS but MDA alone is not associated with MS. ${ }^{14}$ Erythrocyte GPxand CAT activity was increased but slight decrease in SOD activitywasfoundinMSascomparedtoNMS.Superoxide dismutase (SOD) destroys free radical superoxides by converting it to hydrogen peroxide that is further decomposed by CAT or GPx. CAT plays an important role in the acquisition of tolerance to oxidativestress in adaptive response of cells. Since active GPx activity is dependentonGSHconcentrationincreasedGPxactivity causes decreased reduced glutathione. Two vitaminic antioxidants $\alpha$-Tocopherolandascorbateactinsynergism in the membrane and cytosol of the cell. $\alpha$-Tocopherol scavenges lipid peroxy free radicals and interrupts the chain reaction oflipid peroxidationbecomingoxidized itself in the process. Ascorbate present in the aqueous compartments(e.g.cytosol,plasmaandotherbodyfluids) actasawatersolublechain-breakingantioxidant,convert thetocopheroxylradicalbacktoactive $\alpha$-Tocopherol,there by replenishing antioxidantactivity of $\alpha$-Tocopherol. ${ }^{15}$ Thehealthysubjectschoseninthisstudytakingadequate dietmaybehelpingtomaintainnormalhomeostasisin relationtooxidativestressposedbutsedentarylifestyle contributingtometabolicsyndrome.Alargerpopulation studyisneededtofindcorrelationofoxidativestresswith individual components of MS.

\section{CONCLUSION}

In conclusion, although prevalence of MS is higher but oxidativestressisnotsignificantlyincreasedsuggesting that oxidative stress does not contribute much in the pathogenesis of MS in otherwise healthy subjects.

\section{ACKNOWLEDGEMENT}

WearethankfultoResearchGrantCommittee,B.P.Koirala Institute of Health Sciences and Clinical Biochemistry Laboratoryfortheirsupporttoaccomplishthisproject. 
Table 1. Distribution of MS on the basis of sex, ethnicity and other personnel habits

\begin{tabular}{|c|c|c|c|c|}
\hline & & $\begin{array}{c}\text { Non Metabolic } \\
\text { syndrome }(n=72)\end{array}$ & Metabolic syndrome $(n=46)$ & $p$ value \\
\hline \multirow{3}{*}{ Sex } & Male & 58 (49.15\%) & 40 (33.89\%) & \multirow{3}{*}{0.36} \\
\hline & & & & \\
\hline & Female & $14(11.86 \%)$ & $6(5.08 \%)$ & \\
\hline \multirow[b]{2}{*}{ Ethnicity } & Aryan & 68 (57.62\%) & 39 (33.05\%) & \multirow[b]{2}{*}{0.07} \\
\hline & Mongolian & $4(3.38 \%)$ & $7(5.93 \%)$ & \\
\hline \multirow{3}{*}{ Life Style } & Sedentary & 12 (10.16\%) & 16 (13.55\%) & \multirow{3}{*}{0.02} \\
\hline & & & & \\
\hline & Non Sedentary & 60 (50.84\%) & $30(25.42 \%)$ & \\
\hline \multirow{3}{*}{ Exercise } & Yes & $60(50.84 \%)$ & 32 (27.11\%) & \multirow{3}{*}{0.07} \\
\hline & & & & \\
\hline & No & 12 (10.16\%) & 14 (11.86\%) & \\
\hline \multirow{3}{*}{ Diet } & Vegetarian & $10(8.47 \%)$ & $3(2.54 \%)$ & \multirow{3}{*}{0.21} \\
\hline & & & & \\
\hline & Non-vegetarian & $62(52.54 \%)$ & $43(36.44 \%)$ & \\
\hline \multirow{2}{*}{ Smoking } & Smokers & $8(6.77 \%)$ & $8(6.77 \%)$ & \multirow{2}{*}{0.33} \\
\hline & Non Smokers & 64 (54.23\%) & 38 (32.20\%) & \\
\hline \multirow{2}{*}{ Alcohol } & Alcoholics & 26 (22.03\%) & 24 (20.33\%) & \multirow[b]{2}{*}{0.08} \\
\hline & Non alcoholics & 46 (38.98\%) & 22 (18.64\%) & \\
\hline
\end{tabular}

Table 2. Comparison of components of MS and other biochemical parameter in NMS and MS

\begin{tabular}{|c|c|c|c|}
\hline Parameters & $\begin{array}{l}\text { Non-metabolic syndrome } \\
\text { (Mean } \pm \text { SD) }\end{array}$ & $\begin{array}{l}\text { Metabolic Syndrome } \\
\quad(\text { Mean } \pm \text { SD) }\end{array}$ & $p$ value \\
\hline Age (year) & $38.11 \pm 6.24$ & $38.28 \pm 6.31$ & 0.885 \\
\hline Weight (kg) & $67.14 \pm 9.89$ & $70.13 \pm 7.12$ & 0.078 \\
\hline Height (cm) & $164.45 \pm 7.18$ & $162.65 \pm 6.74$ & 0.175 \\
\hline Waist (cm) & $88.0 \pm 8.86$ & $95.43 \pm 9.77$ & 0.001 \\
\hline Hip (cm) & $99.18 \pm 8.18$ & $95.80 \pm 12.89$ & 0.084 \\
\hline BMI & $24.79 \pm 3.11$ & $26.59 \pm 3.14$ & 0.002 \\
\hline $\mathrm{SBP}(\mathrm{mmHg})$ & $118.38 \pm 8.21$ & $127.89 \pm 10.55$ & 0.001 \\
\hline DBP (mmHg) & $78.22 \pm 6.51$ & $85.97 \pm 11.79$ & 0.001 \\
\hline Serum Glucose (mg/dl) & $78.01 \pm 12.36$ & $92.91 \pm 19.57$ & 0.001 \\
\hline Serum HDL (mg/dl) & $41.76 \pm 5.44$ & $38.43 \pm 2.79$ & 0.001 \\
\hline Serum Triglycerides (mg/dl) & $156.76 \pm 113.04$ & $223.17 \pm 75.97$ & 0.001 \\
\hline Serum Cholesterol (mg/dl) & $168.40 \pm 30.94$ & $171.37 \pm 41.39$ & 0.657 \\
\hline $\mathrm{Hb}(\mathrm{gm} / \mathrm{dl})$ & $14.65 \pm 1.49$ & $13.09 \pm 1.47$ & 0.001 \\
\hline
\end{tabular}


Table 3. Comparison of Antioxidants and oxidants in NMS and MS group

\begin{tabular}{|c|c|c|c|}
\hline Parameters & $\begin{array}{l}\text { Non metabolic syndrome } \\
\quad(\text { Mean } \pm \text { SD) }\end{array}$ & $\begin{array}{l}\text { Metabolic Syndrome } \\
\text { ( Mean } \pm \text { SD) }\end{array}$ & $p$ value \\
\hline Plasma MDA (nmol/ml) & $3.66 \pm 0.59$ & $3.86 \pm 0.66$ & 0.081 \\
\hline Serum Bilirubin (mg/dl) & $0.20 \pm 0.07$ & $0.23 \pm 0.09$ & 0.116 \\
\hline Serum Albumin (gm/dl) & $4.44 \pm 0.41$ & $4.47 \pm 0.26$ & 0.594 \\
\hline Blood GSH (mg/dl) & $29.67 \pm 4.81$ & $28.38 \pm 2.84$ & 0.102 \\
\hline Plasma Vitamin C (mg/dl) & $1.19 \pm 0.24$ & $1.17 \pm 0.16$ & 0.664 \\
\hline Plasma Vitamin E (mg/dl) & $1.00 \pm 0.11$ & $1.00 \pm 0.15$ & 0.757 \\
\hline Plasma Uric Acid (mg/dl) & $6.03 \pm 1.03$ & $6.51 \pm 1.04$ & 0.014 \\
\hline Erythrocyte GPx (IU/gmHb) & $45.93 \pm 7.31$ & $47.02 \pm 6.12$ & 0.403 \\
\hline Erythrocyte CAT (Unit/mgHb) & $43.74 \pm 7.42$ & $46.73 \pm 5.18$ & 0.018 \\
\hline Erythrocyte SOD(IU/gmHb) & $889.46 \pm 152.04$ & $882.14 \pm 131.69$ & 0.788 \\
\hline
\end{tabular}

\section{REFERENCES}

1. Ford E, Giles W, Dietz W. Prevalence of the metabolic syndrome among US adults. Findings from the Third National HealthandNutritionExaminationSurvey.JAMA 2002;287:356-59.

2. Shrestha S, Das BKL, Baral N and Chandra L. Association ofmetabolicsyndromeanditscomponentswiththyroid dysfunctioninfemales.IntJDiabDevCtreis2007;27:24-26.

3. Pandey S, Baral N, Majhi S, Shrestha S, Das BKL, Chandra $L$ et al. Prevalence of the metabolic syndrome in acute myocardialinfarctionanditsimpactonhospitaloutcomes. Int J Diab Dev Ctreis 2009;29:2;52-5.

4. HigdonJV,FreiB.Obesityandoxidativestress; adirectlink to CVD? Arterioscler Thromb Vasc Biol 2003;23;365-7.

5. YagiK.Lipidperoxideandhumandisease.ChemPhyLipids 1987;5:337-51.

6. Sinha AK. Colorimeter Assay of Catalase. Anal Biochem 1972;47:389-94.

7. Kakkar P, Das B and Viswanathan PN. A modified spectrophotometricassayofsuperoxidedismutase.IndianJ Biochem Biophys 1984;21:130-2.

8. RotruckJT, Pope AL, Ganther HE, Swanson AB:Selenium: Biochemicalrolesasacomponentofglutathioneperoxidase. Science 1973;179:588-90.

9. Beutler E, Duron O, Kelly BM. Improved method for the determination of blood glutathione. J Lab and Clin Med 1963;61:882-8.

10. Sullivan MX, Clarke HCN. A highly specific procedure for ascorbic acid. J Assoc Off Agric Chem 1955; 38:514.
11. Bieri JG, Teets L, Belavculy B, Andrewa EL. Serum vitamin ElevelsinnormaladultpopulationinWashingtonDCarea. Proc Soc Exptl Biol Med 1964; 17: 131-3.

12. DrabkinDL,AustinJM.Spectrophotometricconstantsfor commonhemoglobinderivativesinhuman, dogandrabbit blood. J Biol Chem 1932;98;719-33.

13. Bae JH, Bassenge E, Kim KB, Kim YN, Kim KS, Lee HJ et al.Postprandialhypertriglyceridemiaimpairsendothelial function by enhanced oxidant stress. Atherosclerosis 2001;155:517-23 .

14. RomeroFG,MoranMR.Hypomagnesemia,oxidativestress, inflammationandmetabolicsyndrome.DiabetesMetabRes Rev 2006;22:471-6.

15. WinklerBS, OrselliSM, RexTS.Theredoxcouplebetween glutathioneandascorbicacidachemicalandphysiological perspective. Free Radic Biol Med 1994;17:333-49.

16. Lakka HM, Laaksonen DE, Lakka TA, Niskanen LK, KumpusaloE,TuomilehtoJetal.Themetabolicsyndrome and totalcardiovasculardiseasemortalityinmiddleaged men. JAMA 2002;288:2709-16. 